\title{
Calibration of Accelerometers
}

\section{By Samuel Levy, Albert E. McPherson, and Edward V. Hobbs}

\begin{abstract}
This paper describes three accelerometer calibrators. These calibrators were developed to provide a convenient means for calibrating lightweight accelerometers of the types used for measuring accelerations on airplanes in flight. The first calibrator is a shaking table having a sinusoidal motion with frequencies of 20 to 110 cycles per second and accelerations up to 80 gravity; the second is a portable calibrator producing a known pulse of acceleration with a peak value between 1 and 20 gravity; and the third is a centrifuge, which develops a maximum steady acceleration of about 1,000 gravity.
\end{abstract}

\section{Introduction}

Accelerometers of the types used to measure accelerations on airplanes in flight consist of a mass mounted on springs inside a case. Damping is frequently used to limit the response of the accelerometer at its resonant frequency. The mass is usually guided to make the instrument sensitive to accelerations along one line only. The output of the accelerometer is recorded mechanically for some of the larger instruments. For most of the smaller instruments the output is in the form of changes in an electric circuit, which are recorded on an oscillograph, an oscilloscope, or a pen-and-ink recorder. In the working range, the indicated response of the accelerometer is nearly proportional to the applied acceleration. The factor of proportionality is defined as the calibration factor.

Errors can arise in the accelerometer indication from several sources. The primary source of error is variation in the output with the frequency of the applied accelerations. This may be due to frequency effects in either the accelerometer itself or in the associated recording circuits. Secondary sources of errors are (1) nonlinearity of output with variation of applied acceleration at a fixed frequency due to "stops" that are not properly set or faulty design, and (2) response to accelerations applied in a direction transverse to the axis of the accelerometer due to misalinement of the instrument or inherent design characteristics.

The calibrators described in this paper were developed for the Bureau of Aeronautics, Navy Department, to give information on the calibra- tion factors and on the magnitude of errors in accelerometers intended for airplane use.

\section{Sinusoidal "Shaking Table"}

The "shaking table" calibrator was intended to determine errors in calibration factor due to frequency effects. For this purpose it was essential that the table have a nearly pure sinusoidal motion with a minimum of harmonic distortion over a wide frequency band.

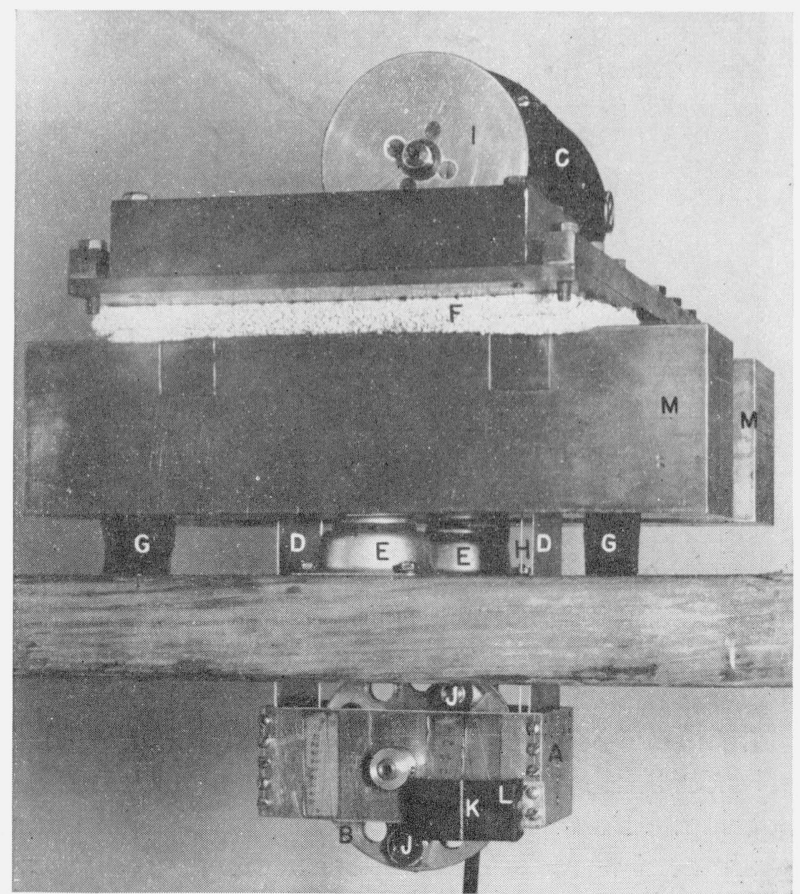

FIgURE 1. Sinusoidal shaking table for calibration of accelerometers. 
The shaking table is shown in figure 1 . It consists of the carriage, $A$, to which the accelerometer is fastened, and means for shaking the carriage, which includes an eccentric disk, $B$, riding with the carriage and a $1 / 2$-hp 8,000-rpm $115-\mathrm{v}$ a-c-d-c series motor, $C$, for rotating the disk over a wide range of speeds.

The carriage, $A$, weighing about $1.2 \mathrm{lb}$, is guided in a nearly straight line in a horizontal direction by flexure plates, $D$. The stiffness of these flexure plates determines the natural frequency of vibration of the table and, therefore, sets a lower limit to the speed range of the table. The natural frequency of the flexure plates, considered as plates clamped at both ends, determines an upper limit to the speed range of the table, beyond which un-

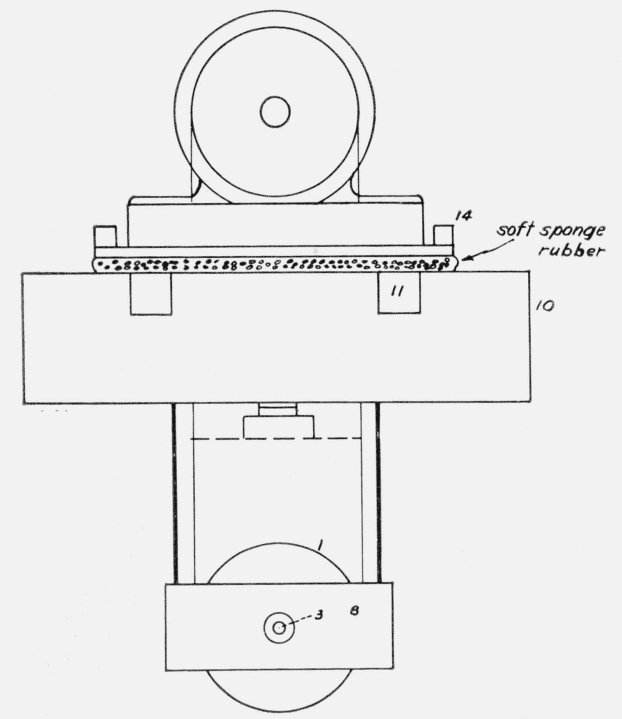

The table is isolated from building vibration by $1 \frac{1}{2}$-in. rubber shock mounts, $E$, and from vibration of the driving motor by a $1 / 2$-in.-thick sponge-rubber shock mount, $F$. In order to prevent coupling between the vibration of the carriage and any nearby flexibly supported masses, the mass of the supports, $M$, was made much larger than the mass of the carriage, $A$, and the shock mounts were chosen to make the natural frequency of the supports $M$ less than $5 \mathrm{c} / \mathrm{s}$. Pieces of sponge rubber, $G$, are used to keep the assembly in balance. Figure 2 is an assembly drawing of the shaking table.

The drive belt, $H$, between the pulley, $I$, and the disk, $B$, figure 1 , is a taut single strand of No. 30 cotton thread. It was found that the small

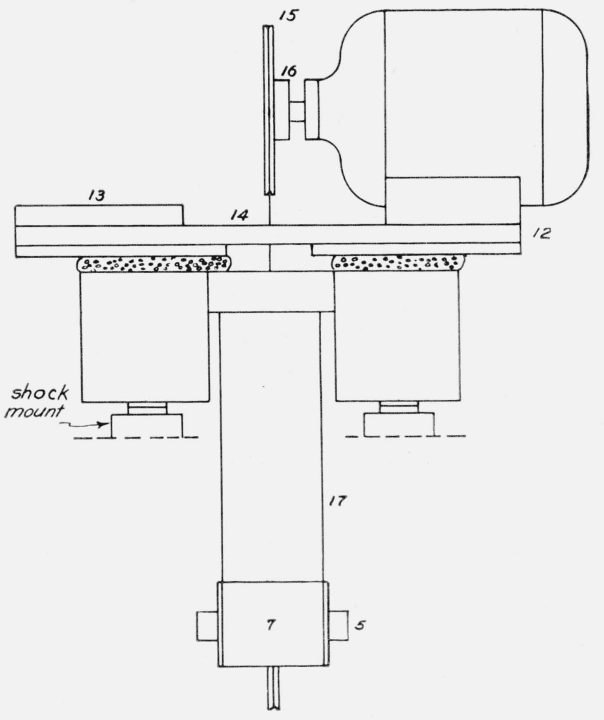

Figure 2. Assembly sheet, shaking table.

Part numbers refer to shop detail drawings (detail drawings available from National Bureau of Standards upon request).

desirable high-frequency vibrations appear in the acceleration output. Four pairs of flexure plates are provided for operating the table over four speed ranges. The table is driven well above its natural frequency in order to make the amplitude insensitive to small variations in the speed of the driving motor. Extraneous high-frequency accelerations due to bearing noise are minimized by holding the eccentric disk, $B$, in a relatively long plain sleeve bearing having a clearance of the order of $0.0003 \mathrm{in}$. in preference to any multipieced bearing. Ball bearings previously used in similar equipment were found to introduce high-frequency accelerations corresponding to the passage of each ball. knot in this thread had no noticeable effect on the acceleration applied by the table.

The eccentric masses, $J$, figure 1 , are standard $1 / 2$-in. bolt-nut combinations when large amplitudes are required and are replaced by specially made small masses when low amplitude is required. The masses are attached to disk $B$ without set screws, since they show no tendency to vibrate loose on the rotating disk, $B$.

\section{Operation}

The accelerometer to be calibrated is attached to the carriage, $A$, figure 1 , with its center line on the center line of the carriage.

The maximum acceleration of the carriage, $A$, 
and the accelerometer attached to it is computed from the formula for harmonic motion,

where

$$
a=14.2 g\left(\frac{S}{1,000}\right)^{2} D
$$

$a=$ peak acceleration

$g=$ acceleration of gravity

$S=$ frequency in cycles per minute

$D=$ double amplitude in inches.

The frequency of vibration, $S$, is measured with a stroboscopic tachometer. The double amplitude, $D$, is measured by observing the extreme positions of a bright spot on the carriage through a stationary telescope having a reticule graduated to 0.01 in. Excellent definition of the extreme positions of motion was obtained by observing a bright 0.001 -in. wire, $K$, figure 1 , illuminated from the side. Good contrast was obtained by placing this wire across the narrow slit in the dull-blackpaper box shown at $L$, figure 1 , and illuminating the wire from a point to the side and slightly below the slit. This measuring system permitted measurement of the double amplit'ide to \pm 0.001 in.

The amplitude of vibration of the carriage is changed by changing the magnitude of the eccentric $J$, in disk, $B$. This is done either by bolting a single mass of suitable size through a hole in the disk or by bolting a pair of equal masses, $M$, through a pair of holes in the disk. The effective unbalanced mass, $m$, of such a pair of masses depends on the angle, $\theta$, subtended by them at the center of the disk and is given by

$$
m=2 M \cos \frac{\theta}{2} .
$$

The bearing in disk $B$ is lubricated with light spindle oil applied to the felt washers at the ends of the disk bearing before each calibration run. Large accelerations at high speeds must not be applied to the carriage, $A$, for too long a period, as they may overheat the bearing, with resulting permanent damage to the close fit of the bearing.

The building up of excessive amplitudes at resonance is avoided either by holding the carriage by hand at the resonant speed or by passing quickly through the resonant speed.

A continuous calibration as a function of frequency is obtained conveniently by recording the accelerometer output on an oscillograph equipped with a time trace. The output of the accelerometer being calibrated is recorded as the shaking table is allowed to coast down from an initial frequency, $S_{0}$, and double amplitude, $D_{0}$. In this case, the frequency, $S$, while coasting, is obtained directly from the record, whereas the corresponding double amplitude $D$ is computed from

$$
D=D_{0} \frac{1-S_{r}{ }^{2} / S_{0}{ }^{2}}{1-S_{r}{ }^{2} / S^{2}}
$$

where $S_{r}$ is the resonant speed of the table. The peak acceleration at any speed, $S$, is then computed by substituting $S$ and $D$ in eq. 1 .

\section{Range}

The double amplitudes obtained during calibration of various instruments with the table are given in figure 3 for each of the four sets of flexure plates and for two or three effective eccentric weights, $m$, in each case. It will be noted that the amplitude with a given set of flexure plates is not exactly proportional to the eccentric weight, $m$, used. The reason for this is that the basic weight of $1.2 \mathrm{lb}$ of the table is substantially increased by the masscs, $m$. The increase is greater when a pair of equal masses, $M$ (eq 2), is used to give the effective mass, $m$, than when a single mass is used. The effective eccentric weight used for a given double amplitude varied also with the weight of the instrument being calibrated and with the frequency. The largest double amplitude obtained was 0.44 in., figure 3 ,a ; the highest frequency was $117 \mathrm{c} / \mathrm{s}$, figure $3, \mathrm{c}$.

The curves of double amplitude versus frequency in figure 3 were converted into the curves of peak acceleration versus frequency, figure 4, by substitution in eq 1 . The largest acceleration obtained was $81 \mathrm{~g}$, figure 4 ,b.

A typical record of acceleration of the carriage as a function of time, obtained with a Statham accelerometer, is shown in figure 5 ,a, and with an NBS vacuum-tube accelerometer, ${ }^{1}$ is shown in figure 5,b. It is evident that the curve of acceleration versus time is nearly sinusoidal.

The acceleration-time curves were practically sinusoidal for all test points shown in figures 3

\footnotetext{
1 Walter Ramberg, J. Research NBS 37, 391 (1946) RP1754.
} 


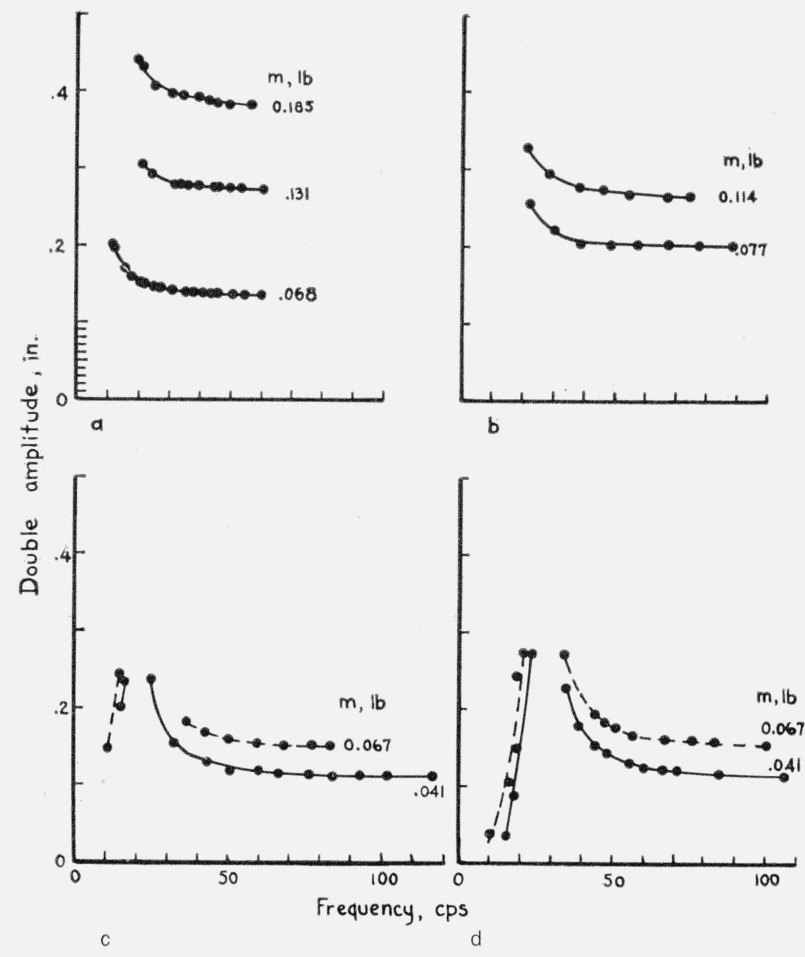

Figure 3. Displacement amplitide vs. frequency for sinusoidal shaking table ( $m=$ effective eccentric weight).

a, 0.030-in. flexure plates; b, 0.037-in. flexure plates; c, 0.053-in. flexure plates; d, 0.064-in. flexure plates.
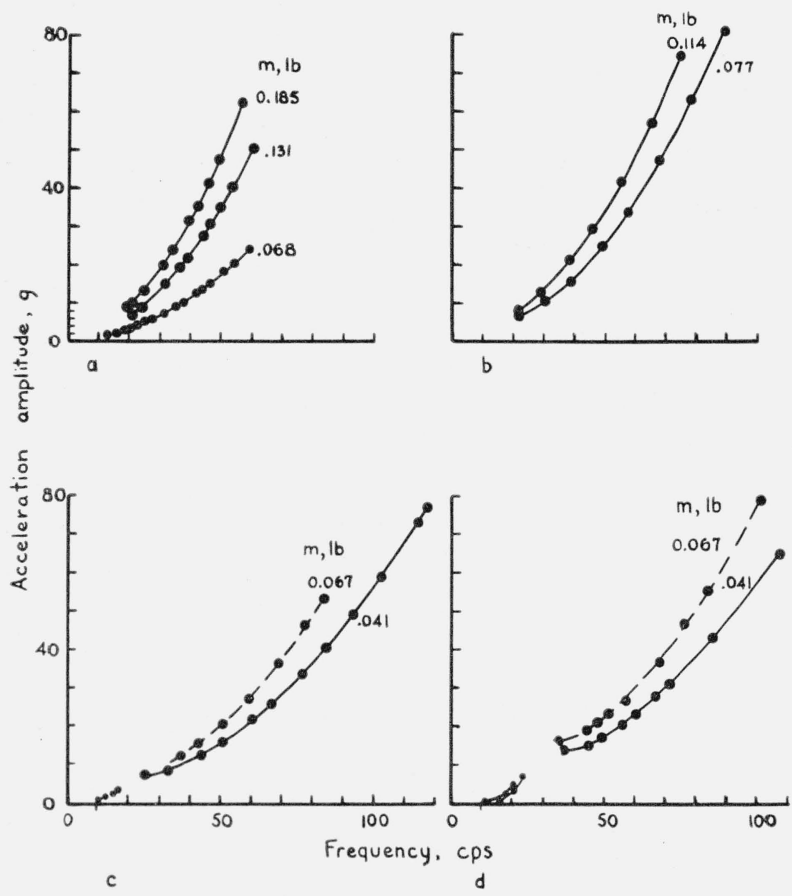

Figure 4. Acceleration versus frequency for sinusoidal shaking table ( $m=$ effective eccentric weight).

a, 0.030-in. flexure plates; b, 0.037-in. flexure plates; c. 0.053-in. flexure plates; d, 0.064-in. flexure plates.

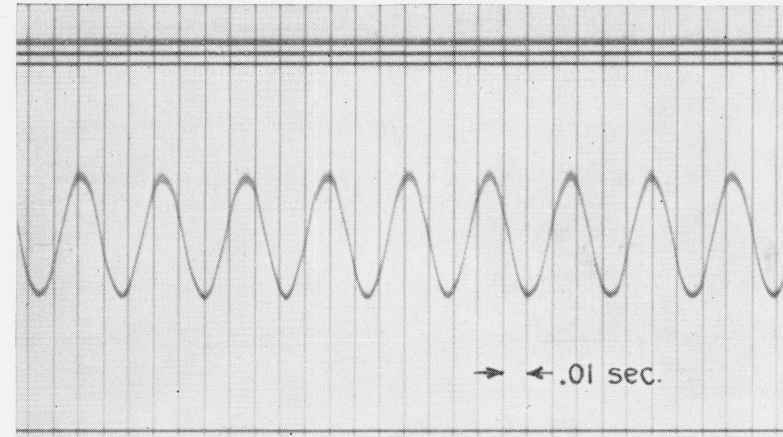

a

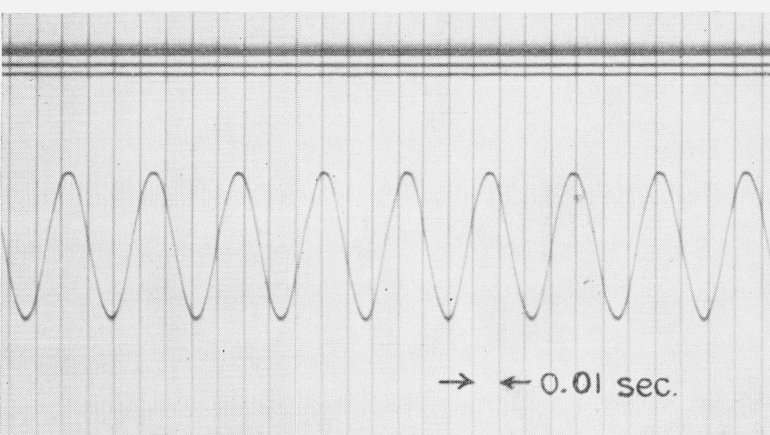

b

Figure 5. Typical records from accelerometers on sinusoidal shaking table.

a, Statham accelerometer, frequency $31 \mathrm{c} / \mathrm{s}$, acceleration amplitude $5.6 \mathrm{~g}$. $b$, NBS vacuum-tıbe acceleration pickup, trequency $31 \mathrm{c} / \mathrm{s}$, acceleration amplitude $6.2 \mathrm{~g}$.

and 4. It was concluded that the shaking table was capable of applying sinusoidal accelerations to accelerometers weighing up to $0.5 \mathrm{lb}$ over a band of frequencies between 20 and $110 \mathrm{c} / \mathrm{s}$.

\section{Portable Calibrator of "Pulse" Type}

The portable calibrator of the "pulse" type was designed for use in the field, where it might be necessary to determine the calibration factor for an accelerometer and its associated electrical circuits just before or after a test. A single spring-mass combination is sometimes used for this purpose; however, it has the disadvantage of subjecting the instrument to a suddenly applied acceleration, which tends to produce resonant response in the instrument and thus prevents it from indicating satisfactorily. In the portable calibrator of the pulse type, the acceleration increases gradually from zero to its maximum value.

The calibrator, figure 6 , is essentially a threemass system consisting of a relatively heavy mass, $M_{1}$, and two relatively light masses, $M_{2}$ and $M_{3}$. 


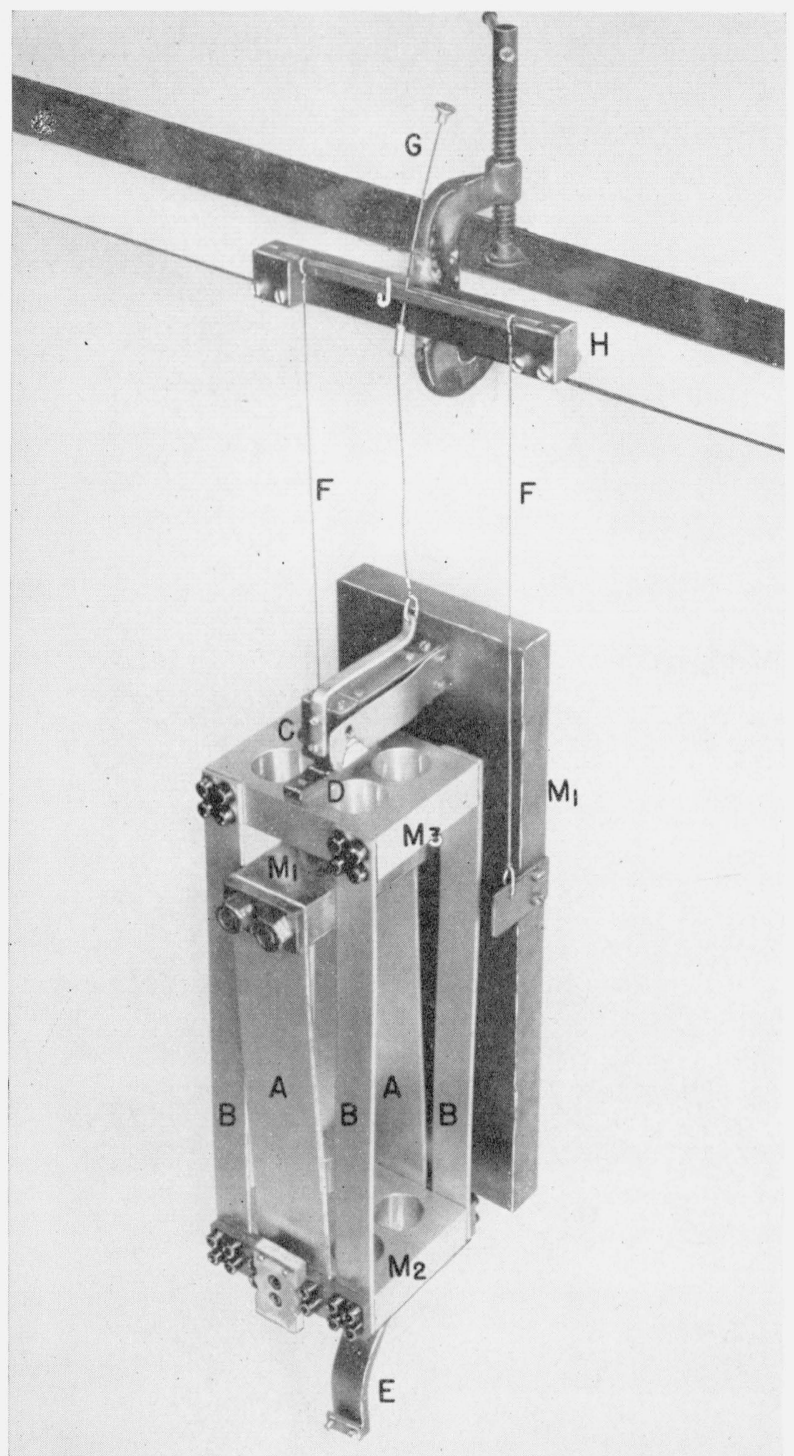

Figure 6. Portable calibrator of "pulse" type for highfrequency accelerometer.

The light mass, $M_{2}$, is flexibly supported by mass $M_{1}$, and mass $M_{3}$ is flexibly supported from $M_{2}$. The acceleration pickups are attached to mass $M_{2}$. They are subjected to a definite pulse of acceleration, rising smoothly from zero when mass $M_{3}$ is suddenly released from a deflected position.

The three masses are connected by double flexure plates $A$ and $B$. Double, rather than single, flexure plates are used, since they prevent rotation of mass $M_{2}$, thereby subjecting all accelerometers on $M_{2}$ to the same pulse of acceleration. The position of $M_{3}$ relative to $M_{1}$, before release, is controlled by the point of engagement of the pawl,
$C$, with the seven-position ratchet, $D$. The cable support, $E$, is provided to secure all except the last few inches of accelerometer cable to the heavy mass, $M_{1}$. The whole calibrator is suspended on the wires, $F$, which provide an effective shock mount with negligible damping.

Mass $M_{3}$ is released by drawing the pawl, $C$, with the release wire, $G$, which is guided to minimize its effect on the motion immediately after release.

An assembly drawing of the calibrator is given in figure 7 . The calibrator was originally built for the calibration of vacuum-tube acceleration pickups (see footnote 1), and it is equipped with special clamps for mounting the unmounted pickups on mass $M_{2}$. It has an additional mounting table, figure 7 , part 25, for attaching other pickups.

In assembling the calibrator, all bolts are tightened as much as possible to reduce mechanical damping to a minimum and to insure that no joint will lift clear during vibration, thus causing nonlinear behavior.

Distance $A$, figure 7 , is chosen so that the release pawl engages the lowest tooth on the ratchet when the calibrator is hanging vertically and the springs are not bent. This distance is about $3.0 \mathrm{in}$.

Distance $B$, figure 7 , is chosen so that the release pawl fully engages the highest tooth on the ratchet when the flexure springs, part 14, are not bent. This distance is about $3.3 \mathrm{in}$.

Distance $C$, figure 7 , is chosen so that the hole in the trigger lock, part 15, engages a No. 6-32 filister-head screw in the side of the upper trigger block, part 13, in such a way as to hold the pawl out of contact with the ratchet during vibration of the calibrator. A thin piece of rubber is placed between the trigger lock and the upper trigger block to cushion the closing of the lock and thus prevent the setting up of undesirable high-frequency vibrations. The offset distance, $C$, between the center of the hole in the trigger lock and the center of the filister-head screw in the upper trigger block is about $0.1 \mathrm{in}$. when the pawl engages the lowest tooth of the ratchet.

Distance $D$, figure 7 , is chosen so that the base of the calibrator hangs vertically from the support wires when the release pawl engages the lowest tooth of the ratchet. This distance is about $0.5 \mathrm{in}$. 


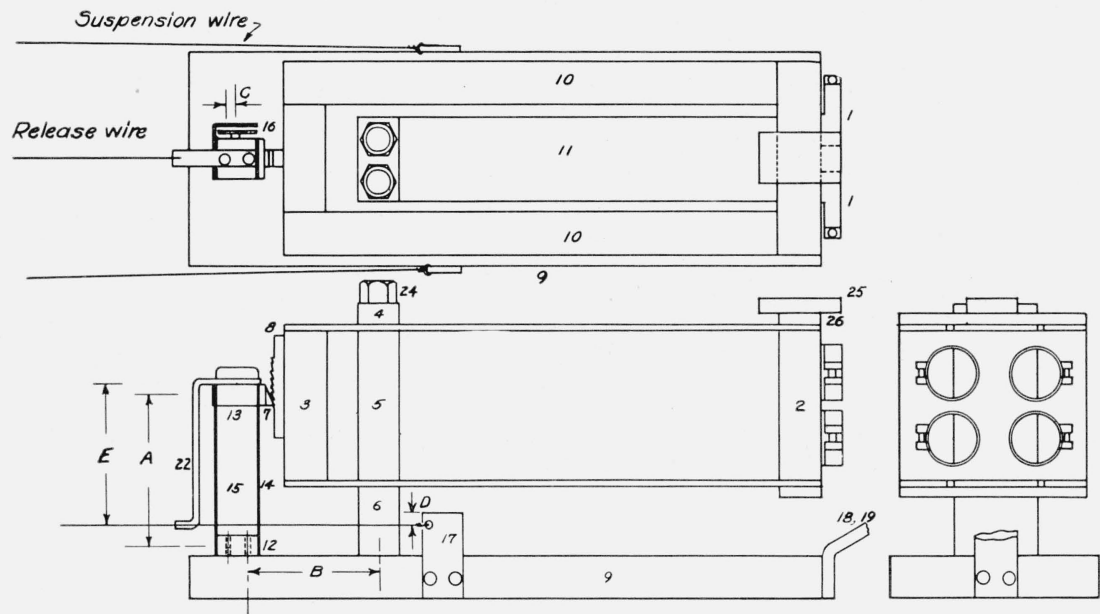

Figure 7. As:embly sheet, pulse calibrator.

Part numbers refer to shop detail drawings (detail drawings available from National Bureau of Standards).

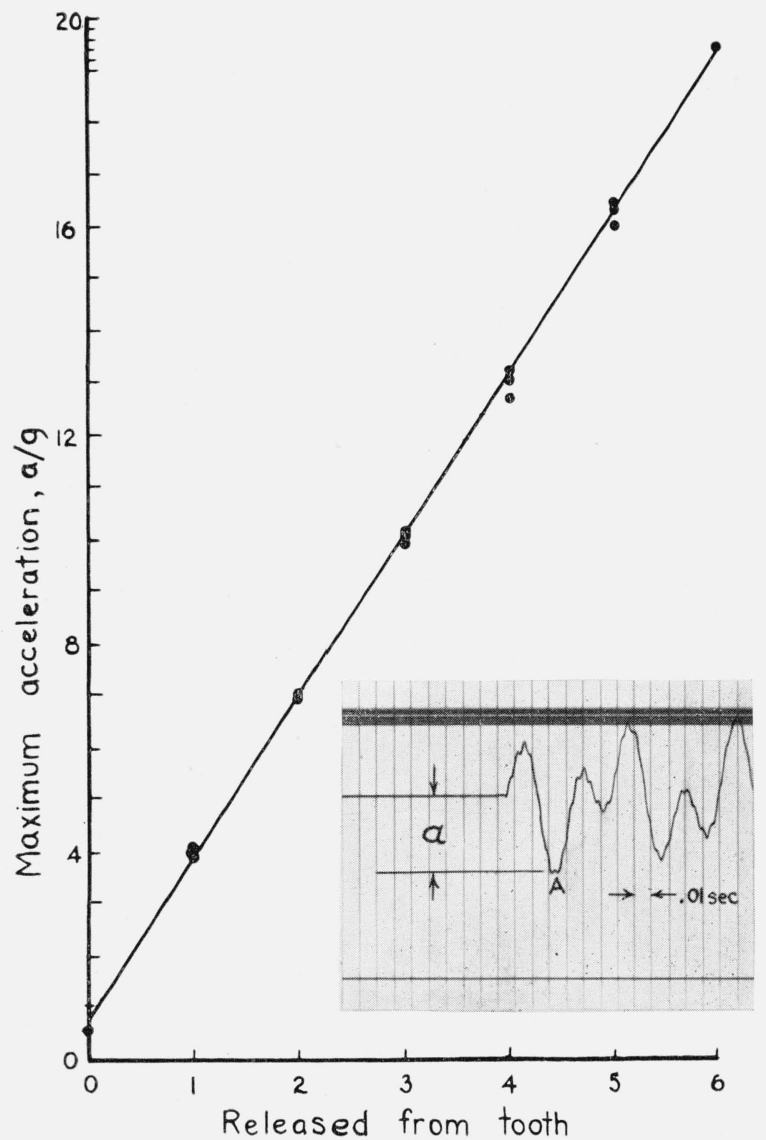

Figure 8. Calibration of pulse-type calibrator using vacuum-tube accelerometer.

Distance $E$, figure 7 , is chosen so that the line of action of the trigger release wire is in the same plane as the support wires. This distance is about 3.1 in.

\section{Operation}

A convenient method of using the pulse calibrator is as follows.

Attach support bracket $H$, figure 6 , to edge of table by means of the C-clamp and suspend calibrator by inserting bar $J$ in the bracket as shown. After checking that the calibrator hangs vertically and clear of obstructions, it is removed from the support bracket so that the accelerometers may be more readily attached to mass $M_{2}$. Any cable connections to the accelerometers are attached to the clamp, $E$, figure 6 , about 6 in. from the instrument. The calibrator is again suspended and "cocked" to the desired notch. When the calibrator has come to rest, the pulse can be initiated by means of the trigger wire, $G$.

A typical acceleration-time curve for the calibrator is shown in the insert in figure 8. Most of the acceleration is produced by vibration of the calibrator in its two principal flexural modes at frequencies of about 12 and $30 \mathrm{c} / \mathrm{s}$. In addition, there is a small superimposed acceleration at about $250 \mathrm{c} / \mathrm{s}$ due to vibration of the springs in their fundamental mode as bars clamped at their ends in the masses, $M_{1}, M_{2}$, and $M_{3}$.

The nominal pulse of acceleration is taken as that produced by the two low-frequency modes only. It is obtained from the trace of acceleration versus time by fairing a curve through the mean points of the high-frequency oscillation. It was decided to remove the high-frequency component in this manner as the calibrator is designed primarily to check accelerometers, which should 
record accelerations with a frequency up to 100 cycles per second but need not record accelerations with a frequency as high as $250 \mathrm{c} / \mathrm{s}$.

The second peak of the acceleration-time curve ( $A$, fig. 8 insert) is taken as the calibrating acceleration. Calculation of the motion of $M_{2}$ on the assumption that $M_{1}$ is much larger than $M_{2}$ or $M_{3}$, and that the spring joining $M_{1}$ and $M_{2}$ has the same stiffness, $k$, as the spring joining $M_{2}$ and $M_{3}$, shows that the acceleration of $M_{2}$ is given by

$$
a=d \frac{k}{\sqrt{4 M_{3}^{2}+M_{2}^{2}}}(\cos A t-\cos B t),
$$

where

$$
\begin{aligned}
a & =\text { acceleration of } M_{2} \\
d & =\text { initial deflection of } M_{3} \\
A^{2} & =\frac{k}{2 M_{2} M_{3}}\left(M_{2}+2 M_{3}\right)+\sqrt{M_{2}{ }^{2}+4 M_{3}{ }^{2}} \\
B^{2} & =\frac{k}{2 M_{2} M_{3}}\left(M_{2}+2 M_{3}\right)-\sqrt{M_{2}{ }^{2}+4 M_{3}{ }^{2}} .
\end{aligned}
$$

When $M_{2}$ and $M_{3}$ are nearly equal, eq 4 shows that for the second peak, the phase relation of the two principal modes is such that a small change in the mass of $M_{2}$, corresponding to a change in the mass of the instrument being calibrated, causes a change in the peak acceleration, which can usually be neglected.

\section{Calibration of Calibrator}

The acceleration of $M_{2}$ at the second peak in the acceleration-time curve for release from a given tooth on the trigger ratchet may be determined with a vacuum-tube accelerometer (see footnote 1) as a secondary standard, using the following procedure:

1. The accelerometer is subjected to a $1-g$ change in acceleration by attaching it to a heavy mass, which is then suddenly released. The output for three consecutive releases of the heavy mass is recorded.

2. Using the same recording equipment, the accelerometer is then moved to the calibrator, and the output for release from each of the ratchet teeth is recorded.

3. Step 1 is repeated.

4. The acceleration of $M_{2}$ at the second peak in the acceleration-time curve, for release from a given tooth on the trigger ratchet, is determined on the assumption that the accelerometer output increases linearly with the applied acceleration and that the output per gram is the average of the six readings obtained in steps 1 and 3 .

Three calibrations of a calibrator were made by using this procedure. The observed accelerations are shown as points in figure 8 , together with a line faired through the points. Only two of the 21 measured points differ from this line by more than $0.2 \mathrm{~g}$. The line indicates a maximum acceleration, corresponding to release from tooth 6 , of $19.5 \mathrm{~g}$ and a minimum acceleration, for release from tooth 0 , of $0.8 \mathrm{~g}$.

The change in maximum acceleration applied by the calibrator, due to a change in the weight of the instrument being calibrated, was measured for the calibrator used above by successively adding 0.1-, 0.3 -, and 0.5 -lb weights to $M_{2}$ and repeating the calibrations as before. These calibrations were carried out only for release from tooth 2 and tooth 5 of the ratchet. The results are shown in figure 9

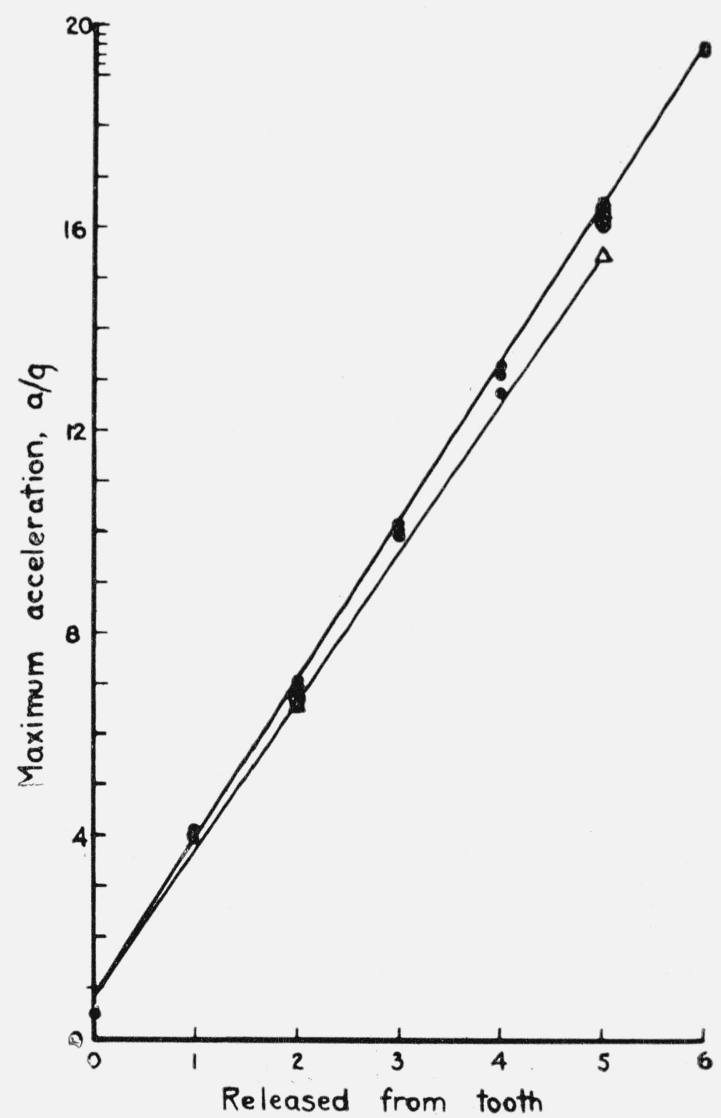

FIGURE 9. Effect of added weight on output of pulse calibrator.

Added weight in pounds $-\bullet, 0 ; \bigcirc, 0.1 ; \square, 0.3 ; \triangle, 0.5$ 


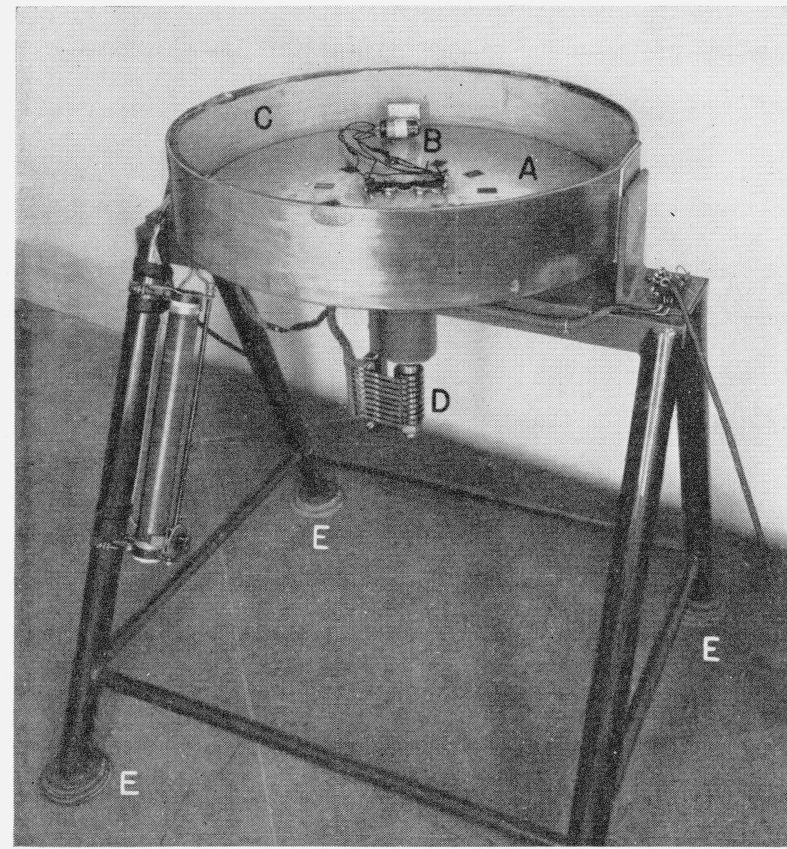

Figure 10. Centrifuge for calibration of accelerometers.

together with points taken from figure 8. The addition of weight to $M_{2}$ caused a decrease in maximum acceleration of the order of 1 percent per $0.1 \mathrm{lb}$ of added weight.

It may be concluded that the portable calibrator of the pulse type may be used in the field for calibration of accelerometers weighing $0.5 \mathrm{lb}$ or less, with a flat response up to at least $30 \mathrm{c} / \mathrm{s}$ and with a range up to about $20 \mathrm{~g}$.

\section{Centrifuge}

The centrifuge was designed for use in the laboratory to give an accurate measure of the calibration factor and to check the linearity between applied acceleration and response up to high sustained accelerations. The centrifuge is shown in figure 10. It consists of a horizontal disk, $A$, which is rigidly attached to a vertical spindle mounted on preloaded ball bearings. The assembly of the spindle is made clear by the sectioned drawing in figure 11.

The spindle is driven with a $1 / 2$-in. V-belt connected to a $1 / 4-\mathrm{hp}$. variable-speed motor (not visible in figure 11). The accelerometer is fastened to disk $A$ at $B$. Electrical connection to the accelerometer is made through the slip rings, $D$. Dynamic balance is maintained by fastening a balance weight an equal distance from the axis of the table opposite $B$. Protection against rupture during test is provided by surrounding disk $A$ with a guard ring, $C$, which is lined with oilbase modeling clay.

The centrifuge is mounted on sponge rubber pads, $E$, to give it a low critical speed, about 5 rps. Disk $A$ will then rotate about its own center of gravity at the operating speeds, well above the critical speed, regardless of deviation from perfect balance.

The table was designed for operation at speeds up to 30 rps. This corresponds to a centripetal acceleration up to $1,000 \mathrm{~g}$ at the periphery of of disk $A$. With suitable attachments, accelerometers weighing $1 \mathrm{lb}$ may be accommodated.

The ball bearings used are Ford part No. B-7065. In assembling the spindle, the bearings are preloaded to the point of slightly restricted rotation by shimming between the inner race of the bearing and the shoulder on the spindle or between the outer race of the bearing and the shoulder on the housing. The bearings must be entirely clean of abrasive matter and lightly oiled.

The slip rings are made of copper, and their brushes are made of copper-graphite, National 543. Both must be kept clean and dry. No lubricant is used on the slip rings.

\section{Operation}

The accelerometer to be calibrated is attached to $\operatorname{disk} A$, and a counterweight of equal mass is attached diametrically opposite and equidistant from the center of the spindle.

The acceleration is computed from the formula,

$$
a=14.2 g\left(\frac{S}{1,000}\right)^{2} D,
$$

where

$a=$ acceleration.

$g=$ acceleration of gravity.

$S=$ speed in revolutions per minute.

$D=$ diameter, in inches, of circle traversed by the center of gravity of the active element of the accelerometer.

The speed of rotation of disk $A$ is measured with a stroboscopic tachometer. It was found helpful to place 10 marks on disk $A$, spaced at equal intervals around the disk. Nine all-black marks and one black with a white line through it 


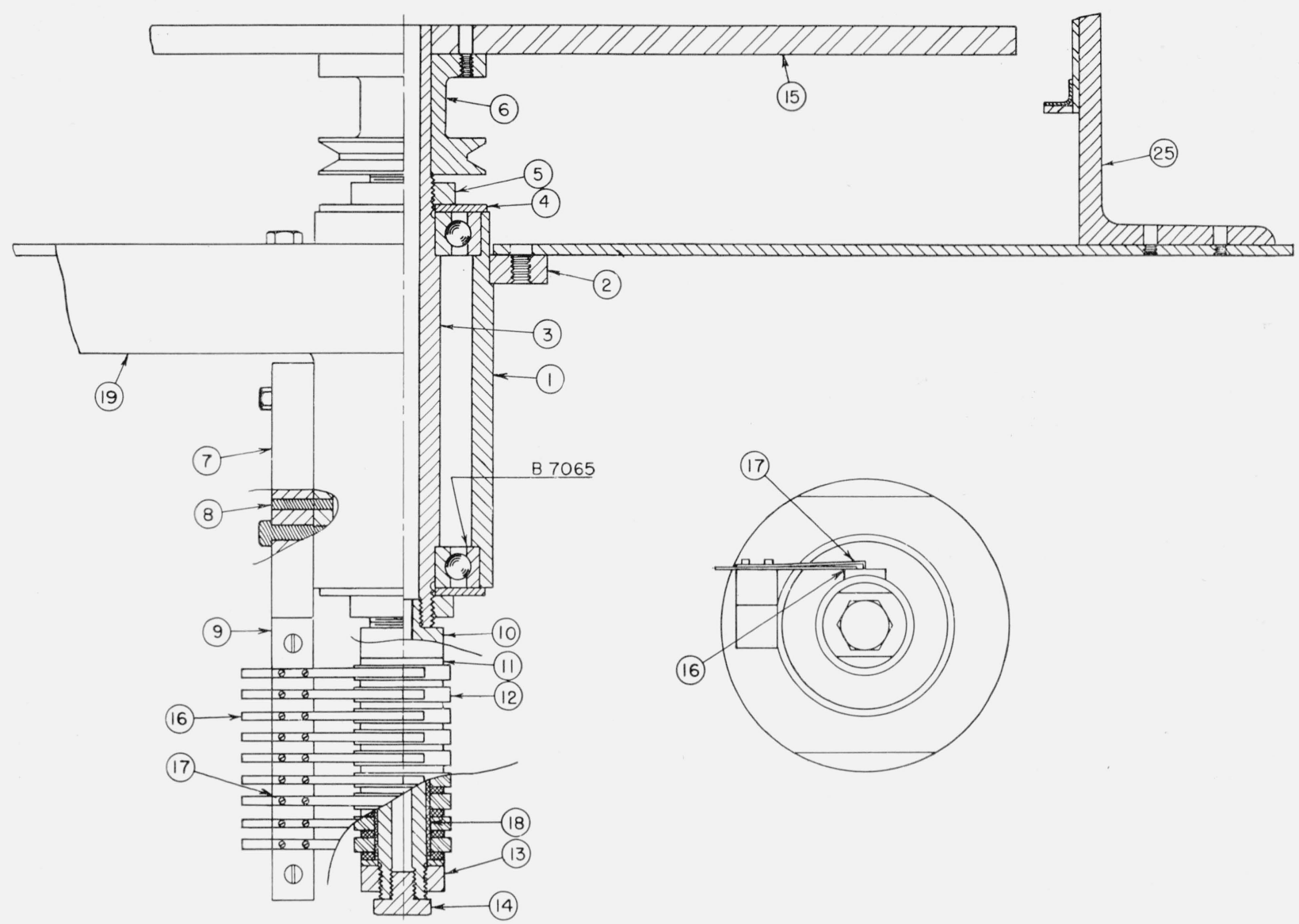

Figure 11. Centrifuge assembly.

Part numbers refer to shop detail drawings (detail drawings available from National Bureau of Standards upon request).

aided in making accurate measurements of the speed of rotation of disk $A$.

\section{Comparison of Results}

The performance of the pulse-type calibrator was compared with that of the sinusoidal shaking table by calibrating the accelerometers, $A$ and $B$. figure 12 , on both devices.

Accelerometer $A$ is an accelerometer of the vacuum-tube type described in the reference given in footnote 1 . It is undamped and has a natural frequency of about 800 cycles per second. Accelerometer $B$ is a Statham accelerometer. It is damped and has a natural frequency of about 350 cycles per second:

The circuits used in each test and the results of the calibration are given in figure 13 for the vacuum-tube accelerometer and in figure 14 for the Statham accelerometer. Inspection of these figures shows that the measured accelerations

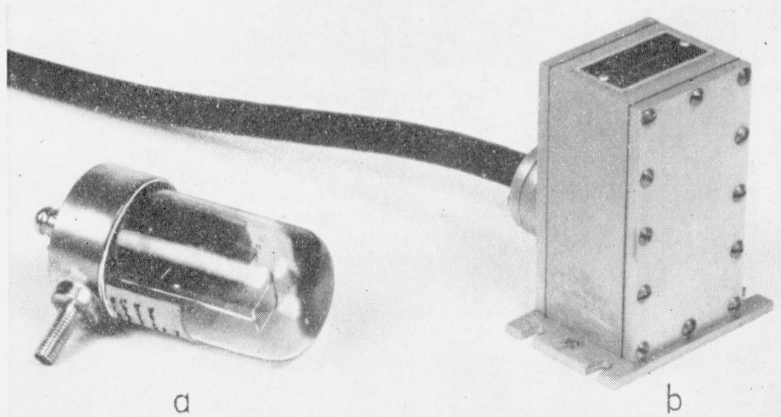

Figure 12. Accelerometers.

a, NBS vacuum-tube accelerometer; b, Statham 12- $g$ accelerometer.

differed less than $\pm 0.5 \mathrm{~g}$ from a common straight line faired through the points.

\section{Check of Calibration Methods}

The procedure for calibrating under steady acceleration on the centrifuge was checked by comparing the recorded output for a vacuum- 


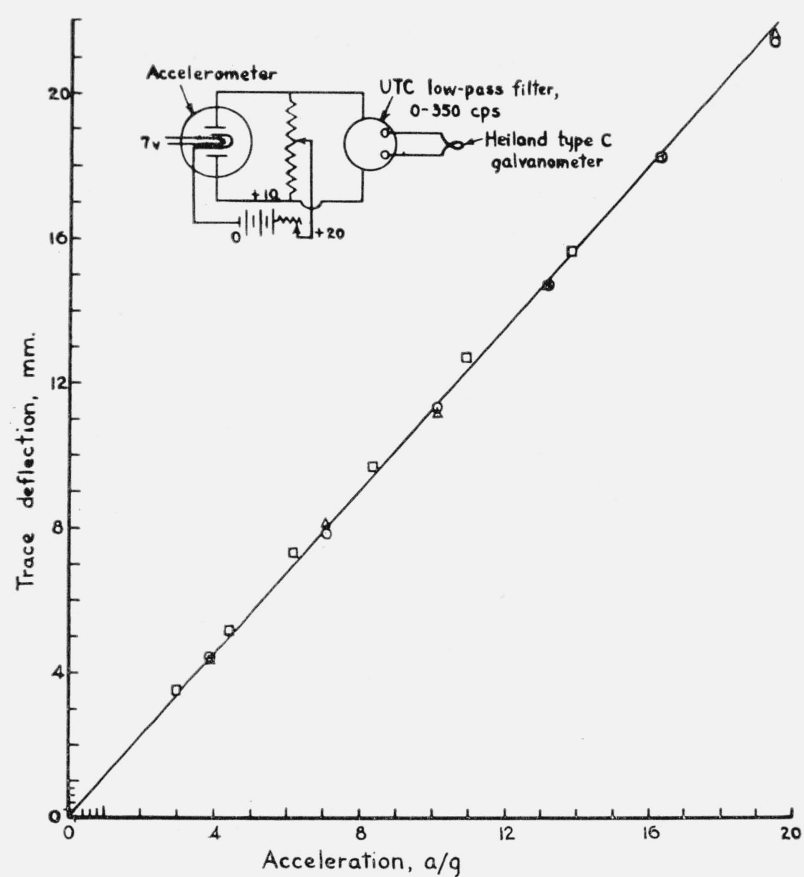

Figure 13. Calibration of vacuum-tube accelerometer. $\bigcirc$, Pulse (first calibration); $\square$, shaking table (seeond calibration) $\triangle$, pulse (third calibration).
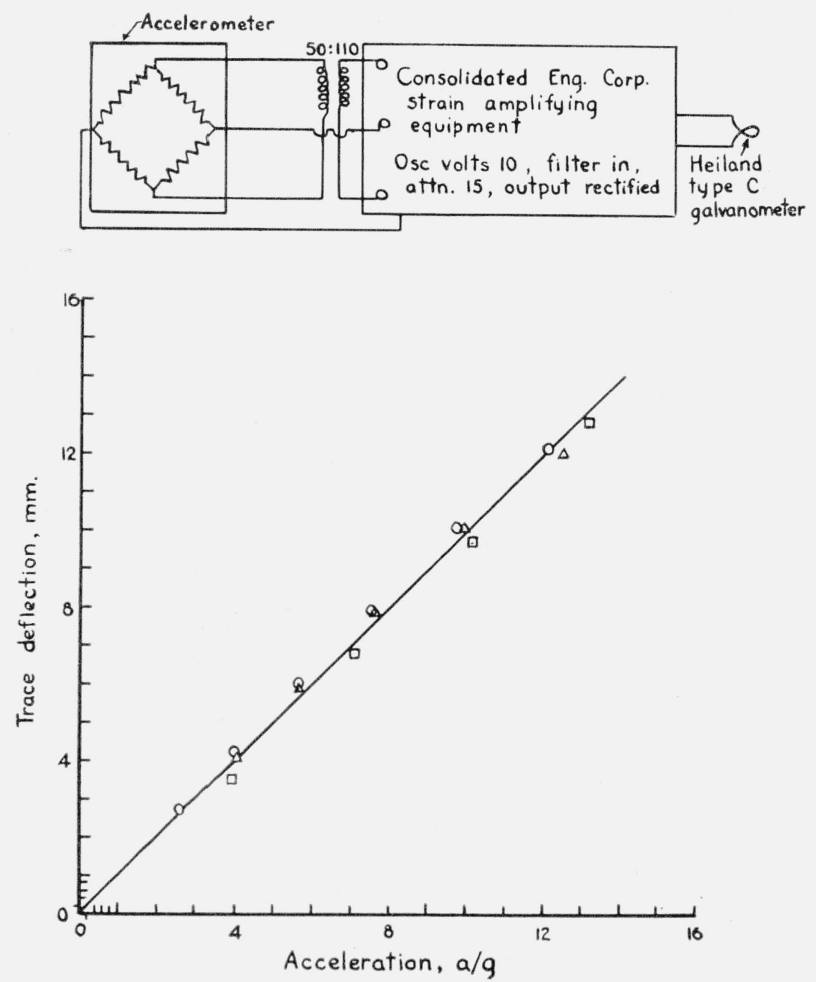

Figure 14. Calibration of Statham accelerometer. $\bigcirc$, shaking table (first calibration); $\square$, pulse (second calibration); $\triangle$ shaking table (thrrd calibration).
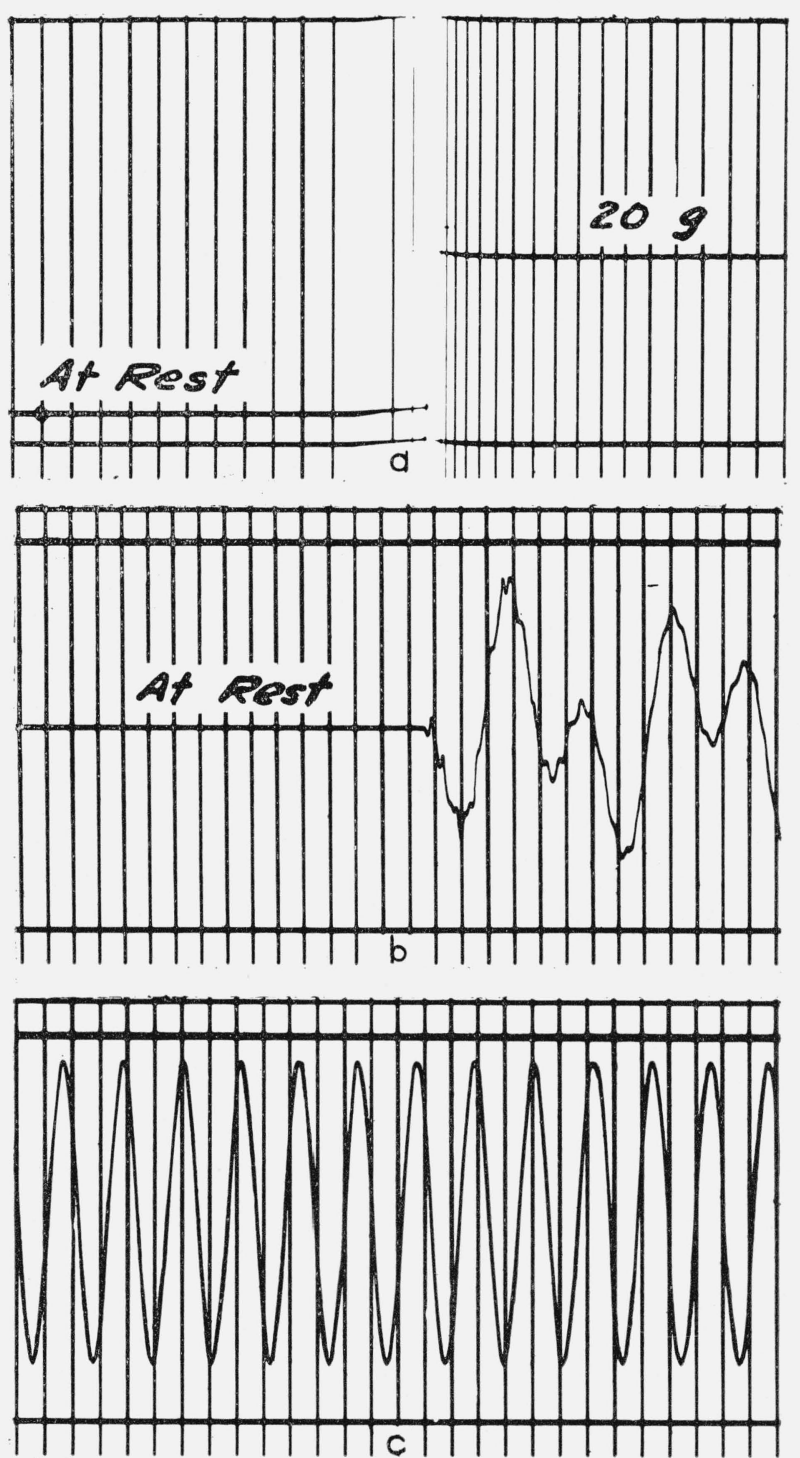

Figure 15. Galvanometer records.

a, Centrifuge, $20 \mathrm{~g}, 0.805 \mathrm{~mm} / \mathrm{g}$; b, pulse calibrator, $19.2 \mathrm{~g}, 0.788 \mathrm{~mm} / \mathrm{g}$; c,

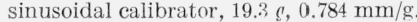

tube accelerometer subjected to about $20 \mathrm{~g}$ on the centrifuge with that obtained on the shaking table and on the pulse-type calibrator. The output was measured with an electric circuit $^{2}$ by using a recorder with a galvanometer of $300 \mathrm{c} / \mathrm{s}$ natural frequency. The records are reproduced in figure 15. It may be seen that the output for $1 \mathrm{~g}$ deviated less than 2 percent from the average value for the three methods.

\footnotetext{
2 Shown in figure 4 of "The measurement of acceleration with a vacuum tube," AIEE Transactions 66 (1947) by Walter Ramberg.
} 


\section{Conclusions}

The sinusoidal calibrator may be used to calibrate accelerometers weighing up to $0.5 \mathrm{lb}$, over a band of frequencies betwecn 20 and $110 \mathrm{c} / \mathrm{s}$. Amplitudes up to $0.4 \mathrm{in}$. may be applied at the low end of the frequency band. Accelerations up to $80 \mathrm{~g}$ may be applied at the high end.

The portable calibrator of the pulse type may be used to check accelerometers with a weight up to $0.5 \mathrm{lb}$ for recording accelerations with a frequency up to at least $30 \mathrm{c} / \mathrm{s}$. The calibrator applies to the accelerometer a known pulse of acceleration with a peak value that may be adjusted in six steps between about 1 and $20 \mathrm{~g}$.
The centrifuge may be used to calibrate accelerometers weighing up to $1 \mathrm{lb}$ with steady acceleration up to $1,000 \mathrm{~g}$.

Acknowledgment is due the Bureau of Aeronau. tics, Navy Department, whose research projects on acceleration pick-ups have provided the impetus and the necessary financial support for the work presented in this paper. The authors take this opportunity to acknowledge also the assistance of members of the Engineering Mechanics Section of the National Bureau of Standards.

Washington, May 10, 1948. 